\title{
Proctite e Infeção Anoretal por Chlamydia trachomatis e Neisseria gonorrhoeae em HSH - Estudo Retrospetivo
}

\author{
João Alves', Jacinta Azevedo², Irene Santo², Maria José Borrego ${ }^{3}$ \\ 'Interno da Formação Específica em Dermatovenereologia/Resident Dermatology and Venereology, Hospital Garcia de Orta, \\ Almada, Portugal \\ ${ }^{2}$ Assistente Hospitalar de Dermatovenereologia, Chefe de Serviço de Medicina Geral e Familiar/Consultant of Dermatology and \\ Venereology, Consultant Chief of General and Family Practitioner, Consulta de Doenças Sexualmente Transmissíveis / Outpatient \\ Clinic of STDs, Centro de Saúde da Lapa, Lisboa, Portugal \\ ${ }^{3}$ Investigadora do Instituto Nacional de Saúde Doutor Ricardo Jorge, Departamento de Doenças Infecciosas, Instituto Nacional de \\ Saúde Doutor Ricardo Jorge/Researcher, Portuguese NIH, Lisboa, Portugal
}

RESUMO - Introdução: As infeções sexualmente transmissíveis (IST) em homens que têm sexo com homens (HSH) têm aumentado nas últimas décadas, nomeadamente as provocadas por Neisseria gonorrhoeae (NG) e por Chlamydia trachomatis (CT), ambas responsáveis por proctites e infecções anorectais. Material e Métodos: Análise retrospetiva da frequência de proctite e infecção anoretal por NG e CT em 2420 HSH observados na Consulta de Doenças Sexualmente Transmissíveis do Centro de Saúde da Lapa, entre Janeiro de 2008 e Dezembro de 2013. São avaliados dados sociodemográficos, comportamentais, clínicos e laboratoriais. Resultados: Foram encontrados sinais e/ou sintomas de proctite em 1,6\% (38/2420) dos HSH, não tendo sido identificado qualquer agente infeccioso em 39,4\% (15/38) destes doentes. Em 38 (16\%) dos 238 HSH a infecção por NG teve localização anoretal; este local anatómico revelou-se infetado por CT em 23 (14,6\%) dos 158 casos positivos, tendo sido identificado o serovar L2b em 7 dos 23 (30,4\%). As infeções anorectais foram mais frequentes em doentes com antecedentes de IST, múltiplos parceiros sexuais e imigrantes. Salienta-se a elevada percentagem de HSH infetados e assintomáticos ou com sinais/sintomas pouco sugestivos de proctite: 55,3\% (21 / 38) e 47,8\%, (11 / 23) dos infetados por NG e CT, respetivamente. Conclusões: A frequência da infeção anoretal assintomática por CT e NG nos HSH que recorrem à consulta DST do CS Lapa é semelhante à descrita para consultas similares noutros países e justifica a necessidade da pesquisa daqueles agentes e de estirpes LGV de CT quando é referido contacto anal recetivo.

PALAVRAS-CHAVE - Chlamydia trachomatis; Doenças do Ânus; Homosexualidade; Infecções por Chlamydia; Neisseria gonorrhoeae; Proctite; Doenças Sexualmente Transmissíveis.

\section{Proctitis and Chlamydia trachomatis and Neisseria gonorrhoeae Anorectal Infection in MSM - A Retrospective Study}

ABSTRACT - Introduction: Sexually transmitted infections (STI) among men who have sex with men (MSM) have increased in recent years, particularly those caused by Neisseria gonorrhoeae (NG) and Chlamydia trachomatis (CT), both responsible for proctitis and anorectal infections. Methods: Retrospective analysis of the frequency of proctitis and NG and CT anorectal infection in 2420 MSM observed in Outpatient Clinic of STD of Lapa Health Center in Lisbon, Portugal, between January 2008 and December 2013. Sociodemographic, behavioral, clinical and laboratory data were assessed. Results: proctitis was identified in 1.6\% (38 / 2420) of MSM.

Trabalho apresentado como Comunicação Oral no Congresso Nacional da SPDV 2014

Oral presentation at the National Congress of the Portuguese Society of Dermatology, 2014.

Correspondência: Dr. João Alves

Serviço de Dermatovenereologia Hospital Garcia de Orta, Almada

Av. Torrado da Silva - 2801-951, Almada, Portugal

E-mail: alves.joaovitor@gmail.com
Recebido/Received

Outubro/October 2015

Aceite/Accepted

Dezembro/December 2015 
In 39.4\% (15/38) of these patients, no infectious agent was identified. In 38 (16\%) of the 238 MSM with NG infection, this agent had an anorectal location; this anatomical site was infected by CT in 23 (14.6\%) of the 158 positive MSM. L2b genotype was identified in 7 of these 23 patients (30.4\%). Anorectal infections were more frequent in immigrants, patients with a previous STI and with multiple sexual partners. The high frequency of MSM with anorectal infection that are asymptomatic or have no signs of proctitis should be emphasized: $55.3 \%(21 / 38)$ and $47.8 \%(11 / 23)$ of patients with NG and CT infection, respectively. Conclusions: The frequency of asymptomatic anorectal infection by CT and NG among MSM in Lapa Health Center is similar to that described in other countries and emphasizes the importance of screening these agents (including LGV strains) in anorectal swabs in MSM with receptive anal intercourse.

KEY-WORDS - Anus Diseases; Chlamydia Infections; Chlamydia trachomatis; Gonorrhea; Homosexuality; Neisseria gonorrhoeae; Proctitis; Sexually Transmitted Diseases.

\section{INTRODUÇÃO}

Os homens que têm sexo com homens (HSH) representam população de risco para a infeção pelo vírus da imunodeficiência humana $(\mathrm{VIH})$ e outras infeções sexualmente transmissíveis (IST). Nas últimas duas décadas tem-se verificado um aumento das taxas de sífilis recente, gonorreia e infecção por Chlamydia trachomatis neste grupo populacional. ${ }^{1,2}$ De forma geral, as proctites sexualmente transmitidas representam uma pequena proporção das infecções anoretais, assumindo, no entanto, uma preponderância maior nos $\mathrm{HSH}$ com práticas de sexo anal receptivo. ${ }^{3}$ Os agentes mais frequentemente associados às proctites sexualmente transmissíveis são Neisseria gonorrhoeae (NG), Chlamydia trachomatis (CT), Treponema pallidum e vírus herpes simplex. ${ }^{3,4}$ Um número considerável destas infecções anoretais são assintomáticas mas, tal como as outras IST, facilitam a aquisição e transmissão do $\mathrm{VIH}$. 5,6 A pesquisa da infecção por CT ou NG nos homens é normalmente efectuada na urina ou no exsudado uretral. Porém, durante a última década, vários estudos têm demonstrado a importância de realizar colheitas orofaríngeas e anoretais na população de $\mathrm{HSH}_{1}{ }^{5}$ pelo que o CDC (Centers for Disease Control and Prevention) recomenda atualmente o rastreio da infeção anoretal por NG e CT nos HSH com antecedentes de sexo anal recetivo no último ano. ${ }^{4}$

O presente estudo retrospetivo pretende avaliar e caracterizar do ponto de vista sociodemográfico, comportamental, clínico e laboratorial os casos de proctite e infecção anoretal por NG e CT em HSH que recorreram à consulta de DST (Doenças Sexualmente Transmissíveis) do Centro de Saúde (CS) da Lapa, entre janeiro de 2008 e dezembro de 2013.

\section{MATERIAL E MÉTODOS}

De janeiro de 2008 a dezembro de 2013 foram observados $2420 \mathrm{HSH}$ (doentes observados pela primeira vez na consulta acrescidos daqueles com novo episódio de IST) e foi efetuada análise retrospectiva dos respetivos processos clínicos, tendo sido avaliadas as variáveis sociodemográficas e comportamentais incluindo: idade; origem geográfica; grau de escolaridade; orientação sexual; número de parceiros sexuais nos últimos 6 meses; prática de sexo anal recetivo; antecedentes de utilização de drogas endovenosas e de IST. Avaliaram-se ainda as manifestações clínicas e a presença de co-infeções à data do diagnóstico.
Para efeitos do presente estudo foram consideradas como infeções sintomáticas (proctite) aquelas que se manifestaram por exsudação anal, fezes mucosanguinolentas, tenesmo e/ ou proctalgia. Na consulta de DST do CS da Lapa, a pesquisa anoretal de NG e CT é efectuada nos indivíduos sintomáticos, nos parceiros sexuais dos doentes com gonorreia ou infecção por $\mathrm{CT}$ urogenital e em HSH assintomáticos com práticas de sexo anal recetivo não protegido.

O diagnóstico laboratorial de gonorreia foi realizado no Instituto Nacional de Saúde Doutor Ricardo Jorge, por cultura de exsudados anais em meio de gelose chocolate PolyViteX VCAT3 (Bio-Mérieux 43611) e posterior identificação das estirpes de NG pelo sistema Vitek ${ }^{\circledR}$ (Bio-Mérieux) ou pelo teste API-NH (Bio-Mérieux 10400). A pesquisa de NG foi também efectuada por PCR (polymerase chain reaction) em tempo real, em simultâneo com a pesquisa de Chlamydia trachomatis, com recurso ao sistema Cobas 4800 (Roche ${ }^{\circledR}$ ) de acordo com as regras do fabricante para amostras de exsudados. A pesquisa do tipo NG-MAST de NG e do genótipo ompA de CT foi efectuada em todas as amostras positivas de acordo com o preconizado pela aplicação informática NG-MAST (http:// www.ng-mast.net//) e com o anteriormente descrito pelo laboratório,7 respectivamente.

\section{RESULTADOS}

A infecção por NG foi identificada em 9,8\% dos HSH (238/2420) e por CT em 6,5\% (158/2420). Setenta e três dos $2420 \mathrm{HSH}(3 \%)$ tinham proctite e/ou infecção anoretal por CT ou NG. A localização anorectal correspondeu a $16 \%$ (38/238) e 14,6\% (23 / 158) das infecções por NG e CT diagnosticadas nos $\mathrm{HSH}$, respectivamente (Tabela 1). A Figura 1 ilustra a percentagem de diagnósticos de infecção anorectal por NG e CT em HSH por ano. Trinta e oito doentes (38 / 73, $52 \%$ ) tinham sinais ou sintomas sugestivos de proctite (Tabela 2). A maioria dos casos ocorreu em indivíduos de nacionalidade estrangeira (42/73) e no grupo etário entre os 20 e 39 anos (65/73, idade média de 30 anos - 19 a 58 anos). Sessenta e oito doentes tinham completado o ensino secundário ou superior. Vinte doentes $(27 \%)$ referiam ter apenas um parceiro sexual nos últimos 6 meses, 38 (52\%) tinham 2 a 4 parceiros e $15(20,5 \%)$ reportavam mais de 4 parceiros sexuais. Vinte e dois indivíduos tinham infeção VIH e 40 (54,8\%) referiam antecedentes de infeções sexualmente transmissíveis. 
Tabela 1 - Características comportamentais e clínicas dos doentes com infecção anorectal por CT e NG

\begin{tabular}{|c|c|c|c|c|c|}
\hline & & $\mathrm{CT}+/ \mathrm{NG}-$ & CT-/NG+ & $\mathbf{C T}+/ \mathbf{N G}+$ & TOTAL \\
\hline \multirow{4}{*}{$\begin{array}{l}N .^{\circ} \text { de parceiros } \\
\text { sexuais }(6 M)\end{array}$} & 1 & 7 & 5 & 3 & 15 \\
\hline & 2 a 4 & 6 & 23 & 0 & 29 \\
\hline & $\geq 5$ & 5 & 5 & 2 & 12 \\
\hline & Total & 18 & 33 & 5 & 56 \\
\hline Antecedentes & Infecção VIH & 4 & 9 & 2 & 15 \\
\hline \multirow{4}{*}{ IST anteriores } & 0 & 10 & 17 & 2 & 29 \\
\hline & 1 & 5 & 11 & 3 & 19 \\
\hline & $\geq 2$ & 3 & 5 & 0 & 8 \\
\hline & Total & 18 & 33 & 5 & 56 \\
\hline \multirow{6}{*}{ Clínica } & Exsudação anal & 5 & 13 & 2 & 20 \\
\hline & Exsudação + ulceração anal & 1 & 0 & 0 & 1 \\
\hline & Ulceração & 4 & 1 & 0 & 5 \\
\hline & Outros & 2 & 4 & 3 & 9 \\
\hline & Assintomáticos & 6 & 15 & 0 & 21 \\
\hline & Total & 18 & 33 & 5 & 56 \\
\hline \multirow{8}{*}{ Co-infecções } & NG uretral & 0 & 4 & 0 & 4 \\
\hline & NG orofaringe & 0 & 3 & 0 & 3 \\
\hline & $\mathrm{CT}$ uretral & 1 & 3 & 0 & 4 \\
\hline & Sifilis recente & 3 & 7 & 0 & 10 \\
\hline & Herpes genital & 2 & 1 & 0 & 3 \\
\hline & $\mathrm{VIHI}$ (de novo) & 4 & 2 & 0 & 6 \\
\hline & Condilomas & 1 & 1 & 0 & 2 \\
\hline & Total & 11 & 21 & 0 & 32 \\
\hline
\end{tabular}

CT, Chlamydia trachomatis; NG, Neisseria gonorrhoeae

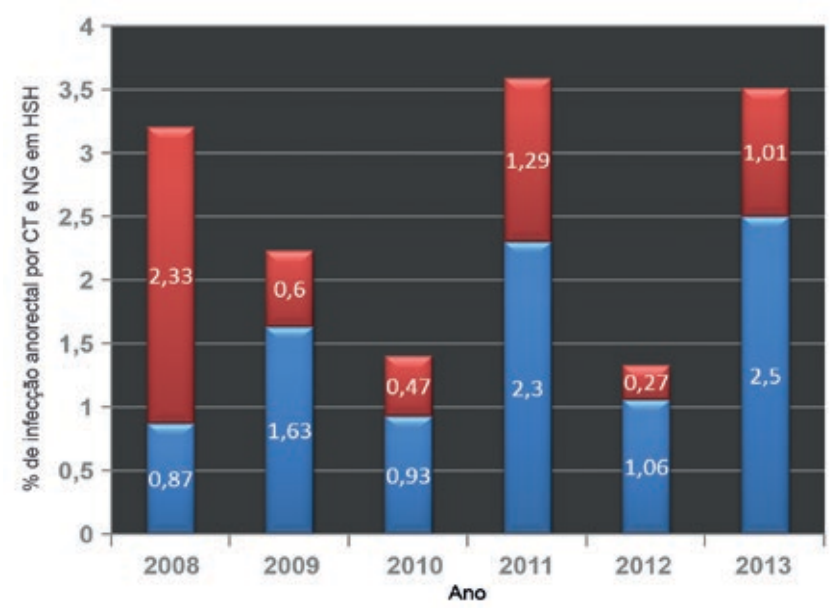

Đinfecçăo por CT घ infecção por NG

Figura 1 - Evolução do número de casos de infecção anorectal por Neisseria gonorrhoeae (NG) e Chlamydia trachomatis (CT) entre 2008 e 2013
Dez doentes (13,7\%) foram referenciados à consulta por parceiros sexuais infetados.

A presença de sinais e sintomas sugestivos de proctite foi observada em 38 dos 2420 (1,6\%) HSH (Tabela 2). Neste grupo de doentes foi identificada infeção por CT em 4 / 38 (10,5\%), NG em 1 1/38 (28,9\%), co-infeção por CT e NG em $2 / 38$ (5,3\%), CT e herpes simplex 1 em $1 / 38$ (2,6\%), CT e sífilis recente em $1 / 38(2,6 \%)$, e NG e sífilis recente em $2 / 38$ $(5,3 \%)$ dos casos. Neste grupo sintomático foram encontradas 8 variedades NG-MAST, predominando os tipos ST2992 e ST3307 (2 casos cada). Num caso foi identificado o tipo ST1407 e noutro o ST3615. No grupo de HSH com proctite foram ainda diagnosticados dois casos de sífilis recente e um caso de infecção pelo VIH de novo. Em 15/38 (39,4\%) dos doentes com proctite não foi possível identificar qualquer etiologia infeciosa (Tabela 2).

Em 7 dos 23 casos $(30,4 \%)$ de infecção anoretal por CT foi identificado o serovar L2b. Destes, 3 eram portugueses, 2 provenientes de países PALOP e 2 brasileiros. Cinco tinham mais de um parceiro sexual nos últimos 6 meses e 3 tinham antecedentes de infecção VIH. Seis eram sintomáticos $(5$ com 
Tabela 2 - Características comportamentais e clínicas dos doentes com diagnóstico de proctite.

\begin{tabular}{|c|c|c|c|c|c|c|c|c|c|}
\hline & & $\mathbf{C T}+/ \mathbf{N G}-$ & $\begin{array}{l}\text { CT-/ } \\
\text { NG+ }\end{array}$ & $\begin{array}{l}\text { CT+1 } \\
\text { NG+ }\end{array}$ & $\begin{array}{l}\text { CT+/ } \\
\text { HS+ }\end{array}$ & $\mathbf{C T}+/ \mathbf{T P}+$ & $\begin{array}{c}\mathrm{NG}+/ \\
\mathrm{TP}+\end{array}$ & TP+ & $\begin{array}{l}\text { Sem agente } \\
\text { identificado }\end{array}$ \\
\hline \multirow{4}{*}{$\begin{array}{l}\mathrm{N} .^{\circ} \text { de } \\
\text { parceiros } \\
\text { sexuais (6M) }\end{array}$} & 1 & 0 & 2 & 1 & 1 & 0 & 0 & 0 & 5 \\
\hline & 2 a 4 & 3 & 9 & 0 & 0 & 0 & 1 & 2 & 7 \\
\hline & $\geq 5$ & 1 & 0 & 1 & 0 & 1 & 1 & 0 & 3 \\
\hline & Total & 4 & 11 & 2 & 1 & 1 & 2 & 2 & 15 \\
\hline Antecedentes & Infecção VIH & 2 & 4 & 0 & 1 & 1 & 0 & 0 & 7 \\
\hline \multirow{4}{*}{ IST anteriores } & 0 & 1 & 4 & 1 & 0 & 1 & 2 & 1 & 3 \\
\hline & 1 & 2 & 3 & 1 & 0 & 0 & 0 & 0 & 7 \\
\hline & $\geq 2$ & 1 & 4 & 0 & 1 & 0 & 0 & 1 & 5 \\
\hline & Total & 4 & 11 & 2 & 1 & 1 & 2 & 2 & 15 \\
\hline \multirow{5}{*}{ Co-infecções } & NG uretral & 0 & 4 & 0 & 0 & 0 & 0 & 0 & 1 \\
\hline & CT uretral & 0 & 1 & 0 & 0 & 0 & 0 & 1 & 0 \\
\hline & $\mathrm{VIHI}$ (de novo) & 0 & 0 & 0 & 0 & 0 & 0 & 0 & 1 \\
\hline & Condilomas & 0 & 0 & 0 & 0 & 0 & 0 & 0 & 1 \\
\hline & Total & 0 & 5 & 0 & 0 & 0 & 0 & 1 & 3 \\
\hline
\end{tabular}

CT, Chlamydia trachomatis; NG, Neisseria gonorrhoeae; HS, Herpes simplex; TP, Treponema pallidum

exsudação anal e 1 com ulceração anal) e apenas um não reportava sintomas. Foram detetadas 3 co-infeções (herpes genital, sífilis recente e infecção urogenital por CT. Nos restantes casos com infecção anoretal por CT foram identificados os genótipos Da (5 casos), G (2 casos) e J (2 casos); em 7 não foi possível determinar a serovariedade.

\section{DISCUSSÃO}

A infecção por NG e CT ocorre mais frequentemente na mucosa urogenital, podendo também afectar as mucosas rectal, orofaríngea e conjuntival. $5,7,8 \mathrm{Nos} \mathrm{HSH}$, a mucosa retal pode ser a única afectada e muitas destas infeções permanecem assintomáticas, favorecendo a sua propagação. ${ }^{5-8}$

No que concerne aos grupos etários mais afectados por proctite e pelas infeções por CT e NG estes são os expectáveis para uma consulta de DST, mais frequentemente procurada por uma população sexualmente activa; de facto, $89 \%$ (65/73) dos doentes tinham entre 20 e 39 anos. Destaca-se o facto de 57,5\% (42/73) dos indivíduos observados não serem naturais de Portugal. Esta percentagem considerável de imigrantes resulta do fluxo migratório na região de Lisboa e do facto de se tratar de uma consulta aberta com fácil acesso aos imigrantes, incluindo os indocumentados. Destaque ainda para a elevada percentagem $(93,1 \%, 68 / 73)$ de doentes que completaram o ensino secundário ou superior, teoricamente uma população com mais fácil acesso a campanhas de prevenção das IST e da infeção $\mathrm{VIH}$. A maioria dos indivíduos tinha antecedentes de IST $(54,8 \% ; 40 / 73)$ e $72,6 \%$ $(53 / 73)$ referiu mais do que um parceiro sexual nos últimos 6 meses. É de realçar que $22 / 73(30,1 \%)$ dos doentes sabiam estar infetados pelo $\mathrm{VIH}$; tais indivíduos, ao adquirirem outras IST revelaram comportamentos de risco (sexo desprotegido) para a disseminação da infeção VIH. Acresce que as IST, nomeadamente a infecção anoretal por NG e CT, facilitam a aquisição e transmissão do $\mathrm{VIH}$ devido à fragilidade da mucosa induzida pela infeção e pelo recrutamento das células dendríticas $^{8}$; de facto, no presente estudo, foi diagnosticada infeção de novo pelo VIH em 13,7\% (7/51) dos casos. É de realçar ainda o elevado número de co-infeções diagnosticadas, destacando-se principalmente a sífilis recente (12/38 $31,6 \%)$, co-infecção uretral ou orofaríngea por NG $(8 / 38$, $21 \%)$ e co-infeção uretral por CT $(5 / 38,13,2 \%)$. Frequências similares de co-infecções foram descritas noutros estudos. ${ }^{9,10}$

Os dados revelados pelo presente estudo mostram uma considerável frequência da infecção por NG e CT em HSH (NG: 9,8\%, 238/2420; CT: 6,5\%, 158/2420). Obviamente, estes dados não são representativos da população portuguesa de $\mathrm{HSH}$, nem mesmo da cidade de Lisboa, dadas as características dos doentes da consulta de DST do CS da Lapa (população jovem, elevado nível educacional, grande percentagem de HSH). Ainda assim, deve destacar-se a maior frequência da infeção por NG $(238 / 2420,9,8 \%)$ em relação à infeção por CT (158/2420, 6,5\%), contrariamente ao descrito pela maioria dos estudos. ${ }^{5,8}$ As infeções genitourinárias por NG e CT predominaram, 84\% (200/238) e 85\% (135/158), respectivamente; a infeção anoretal foi observada em $16 \%$ (38/238) e 14,6\% (23/158) das infeções por NG e CT, respetivamente. Na globalidade, a infeção anoretal por NG e por CT foi detetada em 1,6\% (38/2420) e 1 \% (23/2420) dos $\mathrm{HSH}$, respetivamente (Tabela 1). Comparando estes dados com os de estudos realizados noutros países, verifica-se uma menor percentagem de infeções anoretais por estas bactérias na consulta de DST do CS Lapa. A frequência de infecção anoretal em estudos semelhantes é de 4,6 a 13,2\% para 
NG e de 6 a $14,1 \%$ para CT. ${ }^{5,9-13}$ Estes valores mais elevados podem explicar-se por derivarem de estudos com rastreio sistemático 9,13 ou com pesquisa de CT e NG em todos os HSH que referem sexo anal receptivo no último ano (independentemente do número de parceiros sexuais ou uso de preservativo). ${ }^{5,10-12} \mathrm{Na}$ consulta de DST do CS da Lapa esta pesquisa não é realizada sistematicamente, sendo apenas efetuada nos indivíduos sintomáticos ou com fatores de risco acrescido para a aquisição de infecção anoretal por CT e NG (práticas de sexo anal recetivo não protegido).

Em relação às manifestações clínicas, é de salientar a elevada percentagem de doentes assintomáticos ou com sintomas pouco sugestivos de proctite $(55,3 \%, 21 / 38$ dos doentes com infecção anoretal por NG; 47,8\%, 11/23 dos doentes com infeção anoretal por CT). Mesmo nos cinco doentes com co-infeção anoretal por CT e NG, apenas dois tinham sinais e sintomas compatíveis com proctite (Tabela 2). Conclui-se que se a pesquisa anoretal de CT e NG fosse apenas realizada em doentes com proctite ou com ulceração anal, 53,6\% (30/56) das infeções não teriam sido diagnosticadas e os doentes não teriam sido tratados. Tendo em conta estes resultados, a pesquisa de infecção anoretal por CT ou NG deve ser realizada não só nos casos de proctite mas também nos indivíduos assintomáticos com factores de risco para a sua aquisição, tal como recomendado por outros autores. ${ }^{4-6,10,11}$ Quando comparamos com outros estudos similares, ${ }^{5,9-13}$ se no CS da Lapa a pesquisa de CT e NG fosse realizada em todos os $\mathrm{HSH}$ com antecedentes de sexo anal recetivo (independentemente do uso de preservativo ou do número de parceiros sexuais nos últimos 6 meses), a frequência encontrada poderia ser superior. No nosso estudo, à semelhança de outros, ${ }^{10}$ a infeção anoretal por CT foi mais sintomática que a infeção por NG. Uma das possíveis explicações para este facto pode estar na elevada percentagem $(30,4 \% ; 7 / 23)$ de serotipos L2 identificados em doentes com infeção anoretal por CT que, de acordo com o descrito na literatura ${ }^{14,15}$ estão normalmente associados a infeções sintomáticas. De facto, nos oito doentes com infecção anoretal por CT e com sintomas de proctite, em cinco foi identificado o serovar L2b de CT. Assim, a probabilidade de linfogranuloma venéreo (LGV) em doente com proctite por CT foi considerável, reforçando a necessidade da genotipagem de CT nestes casos clínicos. Também é importante realçar que, por vezes, verifica-se co-infecção por CT serovars não-LGV com CT serovars L1-3. ${ }^{8}$ Desde a década de 1960 até 2003 a detecção de LGV era considerada rara nos países industrializados, no entanto, desde o início do milénio têm sido registados surtos em vários países europeus, incluindo Portugal, principalmente em HSH com a infeção pelo VIH. ${ }^{16.17}$ Dos sete doentes com LGV do presente estudo, três tinham infecção VIH conhecida, seis eram sintomáticos e 3 tinham outras co-infeções (herpes genital, CT uretral e sífilis recente). Apesar de rara, por se tratar de doença potencialmente agressiva, associada a complicações e implicar tratamento diferente, o diagnóstico de LGV deve ser sempre considerado em HSH com sintomas sugestivos de proctite. Contudo, estudos recentes revelam que a pesquisa de LGV não é rotineiramente efetuada em exsudados anais, facilitando a disseminação das estirpes LGV na população HSH. ${ }^{18}$ Estes dados são suportados por outros estudos que estimaram a infecção por CT nos exsudados anais de $\mathrm{HSH}$ entre os 7 e 14,8\% e que implicaram o serovar L2 em cerca de 1/3 dos casos de infeção anal por CT. ${ }^{16,18} \mathrm{Em}$ relação aos serovars não-LGV identificados, à semelhança de outros estudos realizados em $\mathrm{HSH}^{8,9}$ e ao contrário do que se verifica na população em geral e nas infeções urogenitais onde predominam os serovars $E$ ou $F^{7}$ no nosso estudo predominaram os serovars Da, $\mathrm{G}$ e J. Em relação às proctites causadas por NG, à semelhança de outros estudos, foram identificadas uma grande variedade de tipos NG-MAST. ${ }^{19-21}$ Em relação às proctites causadas por NG, à semelhança de outros estudos, foi identificada uma grande variedade de tipos NG-MAST; ${ }^{19-21}$ tal como esperado, dada a elevada capacidade discriminativa da metodologia recomendada pelo ECDC ${ }^{22}$ para a epidemiologia molecular de N. gonorrhoeae, formalmente implementada em Portugal em $2010^{23}$. No presente estudo não foi possível identificar qualquer cluster associado a qualquer rede de contactos sexuais. Contudo, num caso, foi isolado o tipo ST1407, apontado na literatura como responsável por resistências antimicrobianas, incluindo às cefalosporinas de largo expectro ${ }^{19,20} \mathrm{e}$, noutro, foi isolado o tipo ST3615, igualmente implicado na resistência à ciprofloxacina num estudo anteriormente realizado em Portugal. ${ }^{21}$

No grupo de doentes com proctite foi identificada infeção por NG em $11 / 38$ (28,9\%), CT em 4/38 (10,5\%) e co-infeção por CT e NG em 2/38 (5,3\%). Em 15/38 (39,4\%) dos doentes com proctite não foi possível identificar qualquer etiologia infeciosa (Quadro 2). Estes dados estão de acordo com outros estudos que apontam a NG como principal causa de proctite sexualmente transmissível. A percentagem de proctites não específicas, onde não é possível determinar a etiologia, também se encontra dentro dos valores mencionados na literatura ( 11 a 46\%). ${ }^{24,25}$ Para reduzir esta percentagem dever-se-á proceder a exame proctológico meticuloso com recurso a exames laboratoriais microbiológicos e moleculares, sendo ainda, por vezes, necessário para esclarecimento etiológico realizar protoscopia com biópsia. ${ }^{3}$

\section{CONCLUSÕES}

A frequência da infeção anoretal por CT e NG em HSH da Consulta de DST do CS da Lapa é considerável, mas mesmo assim inferior à referida em estudos recentemente publicados. ${ }^{5,8,10-13}$ Este facto, associado à elevada percentagem de infeções assintomáticas, dá ênfase às recomendações internacionais, ${ }^{4,6}$ que propõem a pesquisa da infcção anoretal por NG e CT (incluindo a identificação dos serovars LGV) em todos os $\mathrm{HSH}$ que reportem práticas de sexo anal recetivo, incluindo nos indivíduos assintomáticos. Em HSH com sintomas de proctite, a hipótese de LGV deve ser considerada e iniciada terapêutica após colheita para exame laboratorial. Salienta-se também, mais uma vez, a importância do rastreio e tratamento dos indivíduos assintomáticos na prevenção da transmissão e aquisição da infecção VIH e outras IST. 
Conflitos de interesse: Os autores declaram não possuir conflitos de interesse. Suporte financeiro: $O$ presente trabalho não foi suportado por nenhum subsídio ou bolsa. Direito à privacidade e consentimento escrito: Os autores declaram que pediram consentimento ao doente para usar as imagens no artigo.

Conflicts of interest: The authors have no conflicts of interest to declare. Financing Support: This work has not received any contribution, grant or scholarship. Privacy policy and informed consent: The authors declare that the patient gave written informed consent for the use of its photos in this article.

\section{REFERÊNCIAS}

1. Mayer KH. Sexually transmitted diseases in men who have sex with men. Clin Infect Dis. 2011; 53 Suppl 3:S79-83.

2. Campos R, Rocha N, Baptista A. Infecções sexualmente transmissíveis em homens que têm sexo com homens. Rev Soc Port Dermatol Venereol. 2014; 72:201-10.

3. Voth ML, Akbari RP. Sexually transmitted proctitides. Clin Colon Rectal Surg. 2007; 20:58-63.

4. Workowski KA, Berman S; Centers for Disease Control and Prevention (CDC). Sexually transmitted diseases treatment guidelines, 2010. MMWR Recomm Rep 2010; 59:1110.

5. Peters RP, Verweii SP, Nijsten N, Ouburg S, Mutsaers J, Jansen $\mathrm{CL}$, et al. Evaluation of sexual history-based screening of anatomic sites for Chlamydia trachomatis and Neisseria gonorrhoeae infection in men having sex with men in routine practice. BMC Infect Dis 2011; 11:203.

6. de Vries HJ, Zingoni A, White JA, Ross JD, Kreuter A et al. 2013 European Guideline on the management of proctitis, proctocolitis and enteritis caused by sexually transmissible pathogens. Int J STD AIDS 2013; 25:465-74.

7. Nunes A, Borrego MJ, Nunes B, Florindo C, Gomes JP. Evolutionary dynamics of ompA, the gene encoding the Chlamydia trachomatis key antigen. J Bacteriol 2009; 191: 7182-92.

8. Quint KD, Bom RJ, Quint WG, Bruisten SM, van der Loeff $M F$, Morré SA, et al. Anal infections with concomitant Chlamydia trachomatis genotypes among men who have sex with men in Amsterdam, the Netherlands. BMC Infect Dis $2011 ; 11: 63$.

9. Mejuto P, Boga JA, Junquera M Torreblanca A, Leiva PS. Genotyping Chlamydia trachomatis strains among men who have sex with men from a Northern Spain region: a cohort study. BMJ Open 2013; 3: e002330.

10. Turner AN, Reese PC, Ervin M, Davis JA, Fields KS, Bazan JA. HIV, rectal chlamydia, and rectal gonorrhea in men who have sex with men attending a sexually transmitted disease clinic in a midwestern US city. Sex Transm Dis. 2013; 40:433-8.

11. Dudareva-Vizule S, Haar K, Sailer A, Wisplinghoff H, Wisplinghoff $F$, Marcus $U$, et al. Prevalence of pharyngeal and rectal Chlamydia trachomatis and Neisseria gonorrhoeae infections among men who have sex with men in Germany. Sex Transm Infect 2014; 90:46-51.

12. Gratrix J, Singh AE, Bergman J, Egan C, McGinnis J,
Drews SJ, et al. Prevalence and characteristics of rectal chlamydia and gonorrhea cases among men who have sex with men after the introduction of nucleic acid amplification test screening at 2 Canadian sexually transmitted infection clinics. Sex Transm Dis. 2014; 41:589-91.

13. Taylor M, Newman D, Gonzalez J, Skinner J, Khurana R, Mickey T. HIV status and viral loads among men testing positive for rectal gonorrhoea and chlamydia, Maricopa County, Arizona, USA, 2011-2013. HIV Med. 2015; 16:249-54.

14. Lee DM, Fairley CK, Owen L, Horvath L, Chen MY. Lymphogranuloma venereum becomes an established infection among men who have sex with men in Melbourne. Aust N Z J Public Health 2009; 33:94.

15. Ward H, Alexander S, Carder C, Dean G, French P, Ivens $D$, et al. The prevalence of lymphogranuloma venereum (LGV) infection in men who have sex with men: results of a multi-centre case finding study. Sex Transm Infect 2009; 85:173-5.

16. Gomes JP, Nunes A, Florindo C, Ferreira MA, Santo I, Azevedo J, et al. Lymphogranuloma venereum in Portugal: unusual events and new variants during 2007. Sex Transm Dis 2009; 36:88-91.

17. López-Vicente J, Rodríguez-Alcade D, Hernández-Villalba L, Moreno-Sánchez D, Lumbreras-Cabrera M, Barros-Aguado C, et al. Proctitis as the clinical presentation of lymphogranuloma venereum, a re-emerging disease in developed countries. Rev Esp Enferm Dig 2014; 106:5962.

18. Ward H, Martin I, Macdonald N, Alexander S, Simms I, Fenton $\mathrm{K}$, et al. Lymphogranuloma venereum in the United Kingdom. Clin Infect Dis 2007; 44:26-32.

19. Nguyen D, Gose S, Castro L, Chung K, Bernstein K, Samuel $M$, et al. Neisseria gonorrhoeae strain with reduced susceptibilities to extended-spectrum cephalosporins. Emerg Infect Dis 2014; 20:1211-3.

20. Hielmevoll SO, Golparian D, Dedi L, Skutlaberg DH, Haarr E, Christensen A, et al. Phenotypic and genotypic properties of Neisseria gonorrhoeae isolates in Norway in 2009: antimicrobial resistance warrants an immediate change in national management guidelines. Eur J Clin Microbiol Infect Dis 2012; 31:1181-6.

21. Florindo C, Pereira R, Boura M, Nunes B, Paulino A, Gomes JP, et al. Genotypes and antimicrobial-resistant phenotypes of Neisseria gonorrhoeae in Portugal (20042009). Sex Transm Infect 2010; 86:449-53.

22. European Centre for Disease Prevention and Control. Molecular typing of Neisseria gonorrhoeae - results from a pilot study 2010-2011. Stockholm: ECDC; 2012.

23. Rodrigues JC, Reis L, Cordeiro D et al. Vigilância laboratorial das infeções por Neisseria gonorrhoeae em Portugal, 2004-2013. Bol Epidemiol Observações. 2014; 3. ISSN: 0874-2928 | ISSN:2182-8873.

24. Klausner JD, Kohn R, Kent C. Etiology of clinical proctitis among men who have sex with men. Clin Infect Dis. 2004; 38:300-2.

25. Davis TW, Goldstone SE. Sexually transmitted infections as a cause of proctitis in men who have sex with men. Dis Colon Rectum. 2009; 52:507-12. 